PREPARED FOR THE U.S. DEPARTMENT OF ENERGY, UNDER CONTRACT DE-AC02-76CH03073

PPPL-3733

PPPL-3733

UC-70

Fast Ion Non-Adiabaticity in Spherical Tokamaks

by

V.A. Yavorskij, D. Darrow, V.Ya. Goloborod'ko, S.N. Reznik, U. Holzmueller-Steinacker, N. Gorelenkov, and K. Schoepf

August 2002

$N_{\substack{\text { PRInCETOn PLASMA } \\ \text { PHYSIES LABORATORY }}}^{D}$

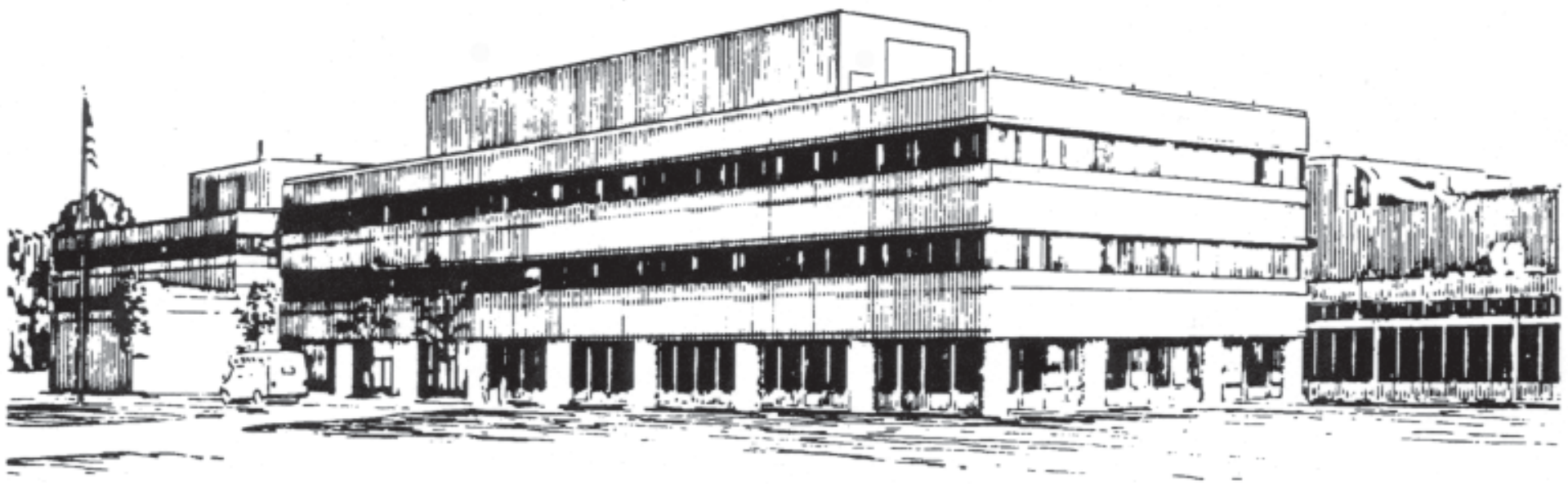

PRINCETON PLASMA PHYSICS LABORATORY PRINCETON UNIVERSITY, PRINCETON, NEW JERSEY 


\section{PPPL Reports Disclaimer}

This report was prepared as an account of work sponsored by an agency of the United States Government. Neither the United States Government nor any agency thereof, nor any of their employees, makes any warranty, express or implied, or assumes any legal liability or responsibility for the accuracy, completeness, or usefulness of any information, apparatus, product, or process disclosed, or represents that its use would not infringe privately owned rights. Reference herein to any specific commercial product, process, or service by trade name, trademark, manufacturer, or otherwise, does not necessarily constitute or imply its endorsement, recommendation, or favoring by the United States Government or any agency thereof. The views and opinions of authors expressed herein do not necessarily state or reflect those of the United States Government or any agency thereof.

\section{Availability}

This report is posted on the U.S. Department of Energy's Princeton Plasma Physics Laboratory Publications and Reports web site in Fiscal Year 2002. The home page for PPPL Reports and Publications is: http://www.pppl.gov/pub_report/

DOE and DOE Contractors can obtain copies of this report from:

U.S. Department of Energy

Office of Scientific and Technical Information

DOE Technical Information Services (DTIS)

P.O. Box 62

Oak Ridge, TN 37831

Telephone: (865) 576-8401

Fax: (865) 576-5728

Email: reports@adonis.osti.gov

This report is available to the general public from:

National Technical Information Service

U.S. Department of Commerce

5285 Port Royal Road

Springfield, VA 22161

Telephone: 1-800-553-6847 or

(703) 605-6000

Fax: (703) 321-8547

Internet: http://www.ntis.gov/ordering.htm 


\title{
FAST ION NON-ADIABATICITY IN SPHERICAL TOKAMAKS
}

\author{
V.A.Yavorskij ${ }^{a}$, D.Darrow ${ }^{b}$, V.Ya.Goloborod'ko ${ }^{a}$, S.N.Reznik ${ }^{a}$, U. Holzmueller-Steinacker ${ }^{c}$, \\ N.Gorelenkov ${ }^{\mathrm{b}}$, K.Schoepf ${ }^{\mathrm{c}}$ \\ ${ }^{a}$ Institute for Nuclear Research, Ukrainian Academy of Sciences, Kyiv, Ukraine \\ ${ }^{\mathrm{b}}$ Princeton Plasma Physics Laboratory, New Jersey, United States of America \\ ${ }^{\mathrm{c}}$ Institute for Theoretical Physics, University of Innsbruck, Austria, Association Euratom-OEAW
}

\begin{abstract}
Transport processes of fast ions in axisymmetric low-aspect-ratio spherical torus (ST) plasmas are investigated, which are induced by the non-conservation of the magnetic moment $\mu$. The reason for non-conservation of $\mu$ of fast ions in ST's is the relatively large adiabaticity parameter $\varepsilon$ typically exceeding the value 0.1 ( $\varepsilon=$ ratio of ion gyro-radius to the gradient scale length of the magnetic field). Both analytical and numerical evaluations of the magnitude of nonadiabatic variations of $\mu$ are performed. Non-adiabaticity effects are shown to be most significant for fast ions for which the bounce oscillations are in resonance with the gyro-motion, i.e. for ions with $\omega_{B}-l \omega_{b}=$ 0 , where $\omega_{B}$ and $\omega_{b}$ represent the bounce averaged gyro-frequency and the bounce frequency, respectively, and $l$ is an integer. The critical threshold of the adiabaticity parameter, $\varepsilon_{\mathrm{cr}}$, to be exceeded for the transition to stochastic behavior of fast ions in axisymmetric ST's is inspected. Nonadiabatic variations of $\mu$ are shown to lead to collisionless transformation of trapped orbits into circulating ones and vice versa. For the case of strong non-adiabaticity, $\varepsilon>\varepsilon_{\mathrm{cr}}$, we assess the transport coefficients describing intense collisionless pitch angle diffusion, whereas, in the case of weak nonadiabaticity, $\varepsilon<\varepsilon_{\mathrm{cr}}$, the more substantial coefficients of enhanced collisional radial diffusion and convection of fast ions gyrating resonantly with the bounce oscillations are estimated.
\end{abstract}

PACS numbers: 52.55.Fa, 52.55.-s, 52.20.Dq

\section{Introduction}

The magnetic moment $\mu=\frac{V_{\perp}^{2}}{2 B}$, where $V_{\perp}$ represents the particle velocity component perpendicular to the magnetic field $\mathbf{B}$ and $B=|\mathbf{B}|$, is proportional to the action variable of Larmor gyration, $E_{\perp} / \omega_{B}$, and hence an adiabatic invariant. It is expected to be well conserved in toroidal axisymmetric fields only in the case of a rather small adiabaticity parameter $\varepsilon$ (ratio of full ion gyro-radius $\rho_{L}$ to the characteristic gradient scale length of the magnetic field B taken here as the major plasma radius $R_{a}$ ). This situation applies to thermals and fast ions confined in conventional axisymmetric tokamaks, where we have $\varepsilon=\rho_{L} / R_{a}<A^{-3 / 2} q^{-1}<<0.1$ [1] with $A$ denoting the plasma aspect ratio and $q$ the safety factor. However, for NBI ions in 
spherical tokamaks (ST's), where typically is $A<1.5$ [2], the adiabaticity parameter is greater than 0.1 and hence the magnetic moment of these ions is not well conserved. The nonadiabatic variations of $\mu$ appear as fast jumps at the time moments $t=t_{s}$ corresponding to the stationary gyro-phase points, $\dot{\vartheta}\left(t_{s}\right)=0$, where $\vartheta$ is the particle gyrophase. Typically, these jumps occur in a rather narrow range of poloidal angles near the outer mid-plane of the torus where the magnetic field is a minimum $[3,4]$. Note that the stationary phase condition $\dot{\vartheta}\left(t_{s}\right)=0$ may also be considered as the local resonant condition $\omega_{B}\left(t_{s}\right)=0$. Since this condition for the toroidal magnetic field is satisfied only in the complex $t$-plane, i.e. when $\operatorname{Im}\left\{t_{s}\right\} \neq 0$, the relative magnitude of these jumps, $\Delta \mu / \mu$, decays exponentially with the inverse adiabaticity parameter, i.e. $\propto \exp \{-\alpha / \varepsilon\}$, where $\alpha=\mathrm{O}(1)$. Already 20-30 years ago a similar exponential decay of $\Delta \mu$ with increasing $\alpha / \varepsilon$ was reported for inhomogeneous magnetic fields of mirror machines and for multipole fields (see, for instance [5-8]), as well as for the magnetic configuration of the earth's magnetosphere [9]; however, none of the results obtained in these early studies is particularly suitable for an axisymmetric toroidal magnetic configuration. We further note that previous investigations of the non-conservation of $\mu$ in toroidal plasmas due to magnetic field ripples $[10,11]$ do not account for the axisymmetric jumps of the magnetic moment as they are considered here. Here both analytical and numerical calculations of the magnitude of non-adiabatic variations of $\mu$ in axisymmetric toroidal magnetic configurations are carried out. Further we discuss fast ion transport processes in spherical tokamaks, which are induced by non-conserved magnetic moments.

\section{Nonadiabatic variations of $\mu$}

Following Refs. [5-8] we start from general expressions for the non-adiabatic jumps of the magnetic moment $\Delta \mu$, which, in the case of $\nabla \times \mathbf{B} \neq 0$, take on the form 


$$
\Delta \mu=-\mu^{1 / 2} \operatorname{Re} \int_{\Delta t} \frac{d t}{B^{1 / 2}}\left\{\frac{2 V^{2}-\mu B}{\rho_{\perp}} \exp (i \vartheta)+\frac{2\left(V^{2}-\mu B\right)}{\rho_{J}} \exp [i(\vartheta-\zeta)]\right\},
$$

where $V$ is the total ion speed, $\rho_{\perp}=\left(|\nabla \ln B|^{2}-\left[(\mathbf{B} \cdot \nabla) B^{-1}\right]^{2}\right)^{-1 / 2}$ denotes the "vacuum" curvature of a field line determined by $\nabla_{\perp} B$, and $\rho_{J}=B^{2} /|\mathbf{B} \times \nabla \times \mathbf{B}|=B^{2} /|\nabla P| \sim r / \beta$ is the current induced curvature which, in the case of equilibrium ( $r$ designates the flux surface radius), is inversely proportional to the pressure gradient $\nabla P$ and, respectively, to the total plasma beta $\beta$ which can reach a few tens of percents in ST's; further, $\vartheta=\vartheta_{0}+\int_{t_{0}}^{t} d t \omega_{B}$ and $\zeta$ the phase shift with $\operatorname{tg} \zeta=(\mathbf{B} \times \nabla B) \cdot(\mathbf{B} \times \nabla \times \mathbf{B}) / B \nabla B \cdot(\mathbf{B} \times \nabla \times \mathbf{B})$. We base our calculations on the analytical model of an axisymmetric toroidal magnetic field $\mathbf{B}(R, Z)$ given in [12]; there the prescribed flux surfaces are determined by a parametric dependence on $R=R(\chi, r, \Pi(r))$ and $Z=Z(\chi, r, \Pi(r))$ where $R$ and $Z$ represent the spatial variables of the cylindrical coordinate system $[R, Z, \varphi], r$ is the flux surface radius, $\chi$ is the poloidal angle, and $\Pi(r)=\{\Delta(r), k(r), \Lambda(r)\}$ denotes a parameter set containing Shafranov shift $\Delta$, elongation $k$ and triangularity $\Lambda$ [12]. The toroidal and poloidal components of the magnetic field are then given by

$$
\mathbf{B}_{t} \equiv J \nabla \varphi=Y(r, \chi) /\langle Y\rangle \nabla \Phi \times \nabla \chi \quad \text { and } \quad \mathbf{B}_{p}=q^{-1}(r) \nabla \varphi \times \nabla \Phi
$$

where the total poloidal current outside a considered flux surface, $J(r)$, and the toroidal magnetic flux $\Phi(r)$ meet the relations

$$
\begin{gathered}
d \Phi / d r=J\langle Y\rangle, Y(r, \chi):=\{R, Z\} / R,\langle\ldots\rangle \equiv \frac{1}{2 \pi} \int d \chi(\ldots) \\
B=|\mathbf{B}|=(J / R) \sqrt{1+\langle Y\rangle^{2} g^{11} / q^{2}}
\end{gathered}
$$

with $g^{11}=\nabla r \cdot \nabla r$ representing the contravariant component of the metric tensor. 
Because the radial and poloidal coordinates $r$ and $\chi$ are slowly varying variables in comparison to the fast gyro phase $\vartheta$, i.e. $\dot{\vartheta} \sim \omega_{B} \gg\{\dot{\chi}, \dot{r} / r\} \sim \omega_{b} \sim \varepsilon \omega_{B}$, the integrals of Eq. (1) can be evaluated using the method of steepest-descent and deforming the integration contour into the upper-half of the complex $t$ plane where it is assumed to pass near the stationary phase points $t=t_{s}$ determined by $\dot{\vartheta}=\omega_{B}=0$ or $B\left(t_{s}\right)=0$. Note that these points are also singularities of the integrand in Eq. (1).

The principal contributions to the integral and, consequently, to the non-adiabatic jumps of the magnetic moment, $\Delta \mu$, come from the vicinity of the stationary points $t=t_{s}$. Fig. 1 displays the real and imaginary parts of the stationary points in the complex $\chi$ - plane for a NSTX magnetic configuration with $\beta=40 \%$ (B model I of [12]). As seen, the stationary points $\chi=\chi\left(t_{s}\right) \equiv \chi_{s}$ are located in the vicinity of the midplane $(\chi=0)$ and $\operatorname{Im} \chi_{s}$ decreases with a greater flux surface radius. For $B \rightarrow 0$ the curvature radii can be represented as

$$
\rho_{\perp}^{-1} \cong i B_{0}^{3} \eta_{s}^{3} /\left(3 B^{3}\right)+O\left(B^{-2}\right) \quad \text { and } \quad \rho_{J}^{-1} \cong i q\left|\frac{d P}{d r}\right| /\left(\langle Y\rangle B^{2}\right)+O\left(B^{-1}\right)
$$

where $\eta_{s}=-\left[3\langle Y\rangle\left(\ln g^{11}\right)_{\chi} /(2 q R Y)\right]_{s}^{1 / 3} J /\left(B_{0} R_{s}\right), \quad B_{0}=B(\chi=0)$ and the subscript "s" indicates values at stationary points. Moreover, inspecting the expression for $d B / d t$ in the lowest order drift approximation, it follows that we may approximate $B \cong B_{0} \eta_{s} V^{1 / 3}\left(t-t_{s}\right)^{1 / 3}$. Using the above expansions for curvature radii and $B(t)$, we derive for the non-adiabatic jump corresponding to a single stationary point

$$
\frac{\Delta \mu}{\mu}=-\pi \frac{V}{V_{\perp 0}} \operatorname{Re}\left\{\left[M_{\perp}+M_{J}\right] \exp \left(i \vartheta_{0}-\frac{\alpha}{\varepsilon}\right)\right\}, \frac{\alpha}{\varepsilon}=-i \int_{t_{0}}^{t_{s}} d t \omega_{B}
$$

with 


$$
\begin{aligned}
& M_{\perp}=\Gamma^{-1}(9 / 8) \hat{M} \varepsilon^{-1 / 8}+O\left(\varepsilon^{1 / 8}\right), \quad \hat{M}=\left(-4 i R_{a} \eta_{s}^{3} / 3\right)^{-1 / 8} \\
& M_{J}=4 \Gamma^{-1}(7 / 8) q\langle Y\rangle^{-1} R_{a}|d P / d r| B_{0}^{-2} \hat{M}^{7} \varepsilon^{1 / 8} \exp \left(i \pi / 2-i \varsigma_{0}\right)+O\left(\varepsilon^{3 / 8}\right)
\end{aligned}
$$

For typical tokamak magnetic configurations with weak triangularity, $\Lambda<<1$, and moderate Shafranov shift, $1>|d| / \sqrt{k^{2}-1}>\langle Y\rangle /(q k)$, the factor $\hat{M} \cong\left[\langle Y\rangle\left(k^{2}-1\right) \varepsilon_{t}\left(1-\varepsilon_{t} / d\right)^{3} /\left(4 q d^{2}\right)\right]^{1 / 8}$, in which $d=d \Delta / d r$ and $\varepsilon_{t}=r / R_{0} \equiv r /\left(R_{\mathrm{a}}+\Delta\right)$, is less then or of order unity. Therefore the current term $M_{J}$ and, respectively, the second term of the integrand in Eq. (1) may become important only when $\varepsilon^{1 / 4} \beta \gg\left\langle\langle Y\rangle \varepsilon_{t} / q \approx k \varepsilon_{t}^{2} / q\right.$. Since this condition is not fulfilled in conventional tokamaks nor in spheromaks, we will suppress this current contribution in the following. Let us find now the value of $\operatorname{Re}\{\alpha / \varepsilon\}$ by employing a Taylor expansion of $B(t)$ about the mid-plane, i.e. $B(t)=B_{0}\left[1+\omega_{0}^{2}\left(t-t_{0}\right)^{2}+\mathrm{O}\left(t-t_{0}\right)^{3}\right]$ with $\omega_{0}^{2}=\ddot{B}_{0} /\left(2 B_{0}\right)$. For the stationary point, $t_{s}-t_{0}=i \omega_{0}^{-1}$, we obtain

$$
\operatorname{Re}\left\{\frac{\alpha}{\varepsilon}\right\}=\frac{2}{3} \frac{\omega_{B 0}}{\omega_{0}} \simeq \frac{2}{3} \sqrt{\frac{2 B_{0}^{3}}{B_{\chi \chi 0}}} \frac{\omega_{B 0}}{\left|V_{\|} \mathbf{B} \nabla \chi\right|_{0}} \simeq \frac{2 \sqrt{2} \omega_{B a} R_{a}}{3 V} \frac{V}{\left|V_{\| 0}\right|} \frac{q k \varepsilon_{t}}{\langle Y\rangle} \frac{1}{\sqrt{\varepsilon_{t}\left(1+\varepsilon_{t}\right)}}\left(1+\frac{d \Delta}{d r}\right)(1-\Lambda)
$$

with $\omega_{B a} R_{a}=e J /(m c)$. For $\Lambda \ll 1$ it follows from [12] that $k \varepsilon_{t} /\langle Y\rangle \cong \sqrt{1-\varepsilon_{t}^{2}}$ which, upon insertion in Eq. (7), leads to the qualitative expression

$$
\operatorname{Re}\left\{\frac{\alpha}{\varepsilon}\right\} \cong \frac{2 \sqrt{2} \omega_{B a} R_{a}}{3 V} \frac{V}{\left|V_{\|}\right|} q \sqrt{\frac{R_{a}+\Delta}{r}-1}\left(1+\frac{d \Delta}{d r}\right)(1-\Lambda)
$$

indicating an enhanced non-adiabatic fast particle behavior as toroidicity, Shafranov shift and triangularity are increased. The dependence of $\operatorname{Re}\{\alpha / \varepsilon\}$ on the particle energy was numerically calculated via Eq. (5). This dependence and its analytical approximation as given by Eq. (8) are shown in Fig. 2 for co-circulating deuterons in the NSTX model magnetic field with $\beta=40 \%$ [12]. It is seen that $\operatorname{Re}\{\alpha / \varepsilon\} \leq 6 \div 7$ at the plasma periphery $(r>0.5-0.6 m)$ for circulating $80 \mathrm{keV}$ deuterons. For that the relative non-adiabatic variations of the magnetic 
moment of these deuterons can exceed $10^{-2}$ per bounce period. From Eqs. $(5,6)$ it follows that $\Delta \mu \mu \propto \cos \vartheta_{0}$ and hence, for up-down symmetric orbits $\left(\vartheta_{0}=90^{\circ} \pm 180^{\circ}\right)$, we expect $\Delta \mu \mu=0$. This is also confirmed by Fig. 3 where the numerically obtained relative jumps $\Delta \mu / \mu$ are displayed as a function of $\vartheta_{0}$ for $40 \mathrm{keV}$ co-circulating deuterons in NSTX. The curve shown allows to find the maximum value of these jumps, $\Delta \mu_{\max } / \mu$, per bounce period.

To find the relative jumps $\Delta \mu \mu$ we calculate the orbits of fast ions by solving the Lorentz equation of motion with the 1997 version of LSODAR (Livermore solver for ordinary differential equations) by L. R. Petzold and A. C. Hindmarsh. This solver is a variant version of the LSODE package found in Ref [13]. Use of LSODAR provided the relative accuracy in the conservation of particle energy and toroidal canonical momentum of the order of $10^{-6} \div 10^{-}$ ${ }^{5}$ on the considered time intervals here. Moreover fast ion orbits as well as time variations of the magnetic moment were reproduced with a relative accuracy better than $10^{-5}$ when reversing the direction of time. This accuracy of orbit calculations is reasonable for numerical evaluations of the relative non-adiabatic variations $\Delta \mu / \mu>10^{-4}$ studied in this paper. In Fig. 4 the numerical values $\Delta \mu_{\max } / \mu$ are compared with the analytical maximum non-adiabatic $\mu$ variations given by Eqs. $(5,6,8)$. A reasonable agreement can be seen. The highest values of $\Delta \mu_{\text {max }} / \mu$ are observed at the plasma periphery and, for $80 \mathrm{keV}$ deuterons in a $\beta=40 \%$ NSTX configuration, can reach a few percent per bounce period. Note that these values of $\Delta \mu_{\max } / \mu$ are substantially smaller than those obtained for the numerical EFIT field in NSTX with $\beta=$ 40\%. This is demonstrated in Fig. 5 where the time variations of the bounce-averaged magnetic moment of co-circulating $60 \mathrm{keV}$ deuterons are compared for both cases of (i) analytical $B$ - model and (ii) the EFIT field of $\operatorname{NSTX}(\beta=40 \%)$. The significant enhancement of fast ion non-adiabaticity in the numerical field appears mainly due to a small-scale poloidal modulation of the numerical $B$, the so-called poloidal field ripples [14] arising from 
the strong Shafranov shift of the flux surfaces at the plasma periphery in high beta ST plasmas. Here however we based our calculations on the analytical NSTX $B$-model of Ref . [12] where these poloidal ripples had not been taken into account.

\section{Superadiabaticity $\left(\varepsilon<\varepsilon_{\mathrm{cr}}\right)$ and stochasticity $\left(\varepsilon>\varepsilon_{\mathrm{cr}}\right)$}

Due to the local nature of the non-adiabatic variations of $\mu$ the behavior on times longer then the bounce time can be described by the mapping

$$
\mu-\bar{\mu}=M(\mu) \cos \bar{\vartheta}, \quad \bar{\vartheta}-\vartheta=\Delta \vartheta(\bar{\mu}) \equiv \int_{0}^{\tau_{b}} d t \omega_{B}=2 \pi\left\langle\omega_{B}\right\rangle / \omega_{b},
$$

where $M$ is the magnitude of the change of $\mu$ per bounce period given by Eq. (5) and the bar denotes the values after the jump, i.e. when the particle has crossed the mid-plane. Neglecting the current contribution we derive

$$
M=-\pi \frac{V}{V_{\perp 0}} \mu \operatorname{Re}\left\{M_{\perp} \exp \left[-\operatorname{Im} \frac{\alpha}{\varepsilon}\right]\right\} \exp \left(-\operatorname{Re} \frac{\alpha}{\varepsilon}\right) .
$$

Using the mapping of Eq.(9) yields the stochasticity criterion $K=|M(\mu, \varepsilon) d \Delta \vartheta(\mu, \varepsilon) / d \mu| \geq 1$. Accounting for the exponential dependence $M \sim \exp (-\alpha / \varepsilon)$ and for $\Delta \vartheta(\mu) \sim N / \varepsilon$ it may also be represented by

$$
\varepsilon>\varepsilon_{\text {cr }} \sim \alpha / \ln \left(M_{*}\right), \quad M_{*} \simeq|\hat{M}||d N / d \ln \mu| \sim k q /\langle Y\rangle \gg 1
$$

Taking typical flux surface parameters of spherical tori at the plasma periphery, $r / R_{a} \sim 0.5 \div 0.7, d \Delta / d r \sim-(0.3 \div 0.5), \Lambda \sim 0.3 \div 0.5, k \sim 1.7 \div 2.2$, we find $\varepsilon_{\text {cr }} \geq 0.2 \div 0.3$; hence, for instance, in NSTX one may expect stochastic behavior of $80 \mathrm{keV}$ deuterons, for which $\rho_{L} / R_{a} \geq 0.2$, and non-adiabaticity induced strong collisionless pitch-angle diffusion [3]. In the core plasma where $\varepsilon<\varepsilon_{\mathrm{cr}}$ and $K<<1$, non-adiabatic jumps of the magnetic moment 
will result in the superadiabatic motion of fast ions (superbanana oscillations with $\left.\Delta \mu_{s b} \sim M K^{-1 / 2}, \omega_{s b} \sim K^{-1 / 2} \omega_{b}\right)$ featuring the new invariant

$$
p^{2} / 2-K \sin \vartheta=\text { const } \text { with } \quad p=\left|d \Delta \vartheta / d \mu_{l}\right|\left(\mu-\mu_{l}\right)
$$

derived from Eq. (7) and containing $\mu_{l}$ as the magnetic moment value that corresponds with the resonance number $l$. Typical resonance levels in the plane spanned by the normalized magnetic moment and the maximum radial coordinate are displayed in Fig. 6 for $80 \mathrm{keV}$ deuterons in NSTX. Superbanana oscillations, as given by Eq. (12), can be obtained as well by direct bounce averaging of $\mu$. Time variations of the bounce-averaged magnetic moment in the vicinity of the resonant level $l=10$ can be seen in Fig. 7 together with the corresponding full gyro-orbits in the $(\beta=40 \%)$ numerical EFIT NSTX field.

\section{Non-adiabaticity induced fast ion transport processes}

\section{a) Collisionless transport}

Here we consider specific transport processes of NBI ions in axisymmetric toroidal plasmas, which are induced by the non-conservation of $\mu$ in the absence of collisions. In the phase space domain corresponding to the stochastic regime $\left(\varepsilon>\varepsilon_{\mathrm{cr}}\right)$ non-adiabaticity will obviously effectuate strong pitch angle diffusion at a rate $D_{s t}^{n} / \mu^{2}=(M / \mu)^{2} \omega_{b}$, where $D_{s t}^{n}$ denotes the stochastic diffusion coefficient as caused by the non-conservation of the magnetic moment. For NSTX-like parameters $\left(\omega_{b} \cong 10^{6} s^{-1}, M / \mu \sim 10^{-2} \div 10^{-1}\right)$ this yields $D_{s t}^{n} / \mu^{2} \cong\left(10^{2} \div 10^{4}\right) s^{-1} \gg v_{\perp}$ with $v_{\perp} \sim(1 \div 3) s^{-1}$ representing the Coulomb collisional pitch angle scattering frequency. Evidently, the substantial collisionless variation of $\mu$ will result in enhanced radial diffusion. Note that non-adiabaticity induced radial diffusion is restricted by the conservation of the toroidal canonical momentum. However, at least for circulating ions 
the $P_{\varphi}$-conservation should not blockstrong non-adiabatic radial shifts, $\delta r \geq a$.. Certainly, the variation of the magnetic moment, $\delta \mu$, for $P_{\varphi}=$ const results in a radial shift of the orbit of the order

$$
\delta r \simeq \frac{q \rho_{L}}{\langle Y\rangle} \frac{B \delta \mu}{V V_{\|}} \sim \frac{q \rho_{L}}{k \varepsilon_{t}} \frac{B \delta \mu}{V V_{\|}} ;
$$

hence for $\delta \mu \sim \mu$ the non-adiabaticity induced shift is of the order of the poloidal gyroradius which, in the case of fast ions, can exceed the ST plasma radius.

Another point of interest is that non-adiabaticity may accomplish the collisionless transformation of trapped orbits into circulating ones and vice versa. This effect is demonstrated in Fig. 8 for a $3.5 \mathrm{MeV}$ alpha particle in DTST $(\mathrm{I} / B=10 M A / 2 T)$, which initially occurring as a barely trapped ion (red curve) - is transformed into a marginally circulating one (blue curve) due to non-adiabaticity and not by means of collisions; this process results in a radial displacement of the orbit in the order of $\sim \Delta r_{b}$.

\section{b) Collisional transport}

In the superadiabatic regime $\left(\varepsilon<\varepsilon_{\mathrm{cr}}\right)$ non-adiabaticity leads to additional pitch angle scattering at the rate $D_{\text {coll }}^{n} / \mu^{2}=v_{\perp}\left(\Delta \mu_{s b}^{n} / \mu\right)^{2} f_{r} /\left(\Delta \xi_{r}\right)^{2}$, where $D_{\text {coll }}^{n}$ identifies the resonant non-adiabatic contribution to the collisional pitch angle diffusion coefficient and $\Delta \xi_{r}$ is the scale width in $V_{I} / V$ of the resonance region and $f_{r}$ is the fraction of resonant particles [3]. For NBI ions in NSTX $\left(\Delta \mu_{s b}^{n} / \mu \sim 0.05 \div 0.1, f_{r} \sim 0.2 \div 0.3, \Delta \xi_{r} \sim \Delta\left(V_{\phi} / V\right) \sim 0.02 \div 0.03\right)$ [3] the scattering rate is $D_{\text {coll }}^{n} / \mu^{2} \geq v_{\perp}$.

Since $\omega_{b}$ is dependent on the particle's energy, the slowing down of resonant fast ions will effect radial convection in the superadiabatic regime, $\varepsilon<\varepsilon_{\mathrm{cr}}$. In spite of decreasing energy during slowing down, Fig.9 shows an increase of $\mu$ by $\sim 20 \%$ for resonant $80 \mathrm{keV}$ deuterons in NSTX $(\beta=40 \%)$ decelerating to $71 \mathrm{keV}$. In contrary, $\mu$ of non-resonant particles will be 
reduced by $10 \%$ as apparent from Fig. 10. Further Fig. 9 demonstrates a substantial inward shift $(\Delta R \cong 3 \div 4 \mathrm{~cm})$ of the resonant orbit of a deuteron upon the energy decrease $\Delta E=9 \mathrm{keV}$ and thus indicates a significant radial convection associated with deceleration, which occurs the rate $\Delta R v_{s} E /(a \Delta E) \sim v_{s}$, where $a$ denotes the plasma radius and $v_{s}$ the slowing down frequency. Comparing the slowed down parts of the orbits (represented by blue curves in Figs. 9 and 10) we conclude that the super adiabaticity induced radial shift acts in addition to the radial drift experienced by particles for which the magnetic moment is conserved.

\section{Summary}

Non-adiabatic variations of the magnetic moment are supposed to be crucial to fast ion behavior in axisymmetric toroidal magnetic fields, at least in spherical tokamaks. Nonadiabaticity is shown to be extremely sensitive to the flux surface geometry. Shafranov shift, elongation, triangularity and high toroidicity of the flux surfaces will substantially enhance the non-conservation of the magnetic moment.

In spherical tori, non-adiabaticity of the magnetic moment is expected to lead to strong collisionless pitch angle diffusion of NBI ions in the stochastic domain while it may increase significantly the Coulomb pitch angle scattering rate in the superadiabatic regime. Both pitch angle diffusion mechanisms result in enhanced radial diffusion of fast ions. Moreover, superadiabaticity can induce additional convection of energetic particles gyrating resonantly with bounce oscillations.

\section{Acknowledgement}

This work has been partially carried out within the Association EURATOM-OEAW project P4 and the impact project Investigation of Charged Fusion Product Confinement in JET funded by the Austrian Academy of Sciences under GZ 4229/1-VIII/A/5/2000. The content of the publication is the sole responsibility of its authors and does not necessarily represent the views of the European Commission or its services. 


\section{References}

[1] T.E. Stringer, Plasma Physics, 16 (1974) 651.

[2] J. Spitzer, et al., Fusion Technology, 30 (1996) 1337.

[3] V. A. Yavorskij, et al., Proc. 28th EPS Conf. on Contr. Fusion and Plasma Phys., Madeira, 2001, P5.025; http://www.cfn.ist.utl.pt/EPS2001/fin/pdf/P5.025.pdf

[4] J. Carlsson, Phys. Plasmas, 8 (2001) 4725.

[5] R.J.Hastie, et al., in Plasma Physics and Controlled Nuclear Fusion Research 1968 (Proc. $3^{\text {rd }}$ Int. Conf, Novosibirsk, 1968) Vol. I, IAEA, Vienna (1969) 389.

[6] J. E.Howard, Phys. Fluids, 14 (1971) 2378.

[7] R.H.Cohen, G.Rownalds, J.H.Foote, Phys. Fluids, 21 (1978) 627.

[7] B.V. Chirikov, in Reviews of Plasma Physics, 13, Consultants Bureau, NY (1987).

[9] S.V. Putvinskii, R.V. Shurygin, Sov. J. Plasma Phys., 10 (1984) 933.

[10] J. Buchner, Journal of Geophysical Research, 94 (1989) 11821.

[11] V.A. Yavorskij, et al., Proc. 27th EPS Conf. on Contr. Fus. and Plasma Phys., Budapest, 2000, P1.068; http://sgi30.mki.kfki.hu/EPS2000/eps2000cd/pdf/p1 068.pdf

[12] V.A. Yavorskij, et al., Plasma Phys. Contr. Fusion, 43 (2001) 249.

[13] A.C. Hindmarsh, Odepack, a Systematized Collection of ODE Solvers, in Scientific Computing, R.S.Stepleman et al. (eds.), North-Holland, Amsterdam, 1983, pp. 55-64.

[14] V.A. Yavorskij, et al., Proc. 29th EPS Conf. on Contr. Fus. and Plasma Phys., Montreux, 2002, P4.084. 


\section{Figure captions}

Fig. 1 Real and imaginary parts of stationary points $\dot{\vartheta}=0$ in the complex $\chi$ - plane for different flux surface radii.

Fig. 2 Dependencies of $\operatorname{Re}\{\alpha / \varepsilon\}$ on the particle energy for the NSTX $(\beta=40 \%)$ analytical model field [11] for different flux surface radii $r$.

Fig. 3 Relative variations of the magnetic moment of $40 \mathrm{keV}$ deuterons as varying with the gyrophase $\vartheta_{0}$ at the midplane. Circles account for the numerically obtained values $\Delta \mu / \mu$, while the solid line represents their fit curve.

Fig. 4 Maximum relative variations of the magnetic moment per bounce period as a function of the particle energy.

Fig. 5 Time variations of the bounce averaged magnetic moment of $60 \mathrm{keV}$ deuterons in the analytically modeled and in the numerical EFIT field for $\operatorname{NSTX}(\beta=40 \%)$.

Fig. 6 Resonance levels for $80 \mathrm{keV}$ co-going deuterons in NSTX.

Fig. 7 Oscillations of $\langle\mu\rangle$ of $80 \mathrm{keV}$ deuterons in the vicinity of the $l=10$ resonance level as dependent on the toroidal velocity component, $V_{\varphi} / V$.

Fig. 8 Collisionless transformation of a barely trapped $3.5 \mathrm{MeV}$ alpha particle in DTST $(\mathrm{I} / B=10 M A / 2 T)$ into a marginally circulating one.

Fig. 9 Full gyro-orbit and time variation of $\mu$ of a resonant $(l=10) 80 \mathrm{keV}$ deuteron in NSTX ( $\beta=40 \%$ ) during slowing down to $71 \mathrm{keV}$.

Fig.10 Full gyro-orbit and time variation of $\mu$ of a non-resonant $80 \mathrm{keV}$ deuteron in NSTX $(\beta=40 \%)$ during slowing down to $71 \mathrm{keV}$. 


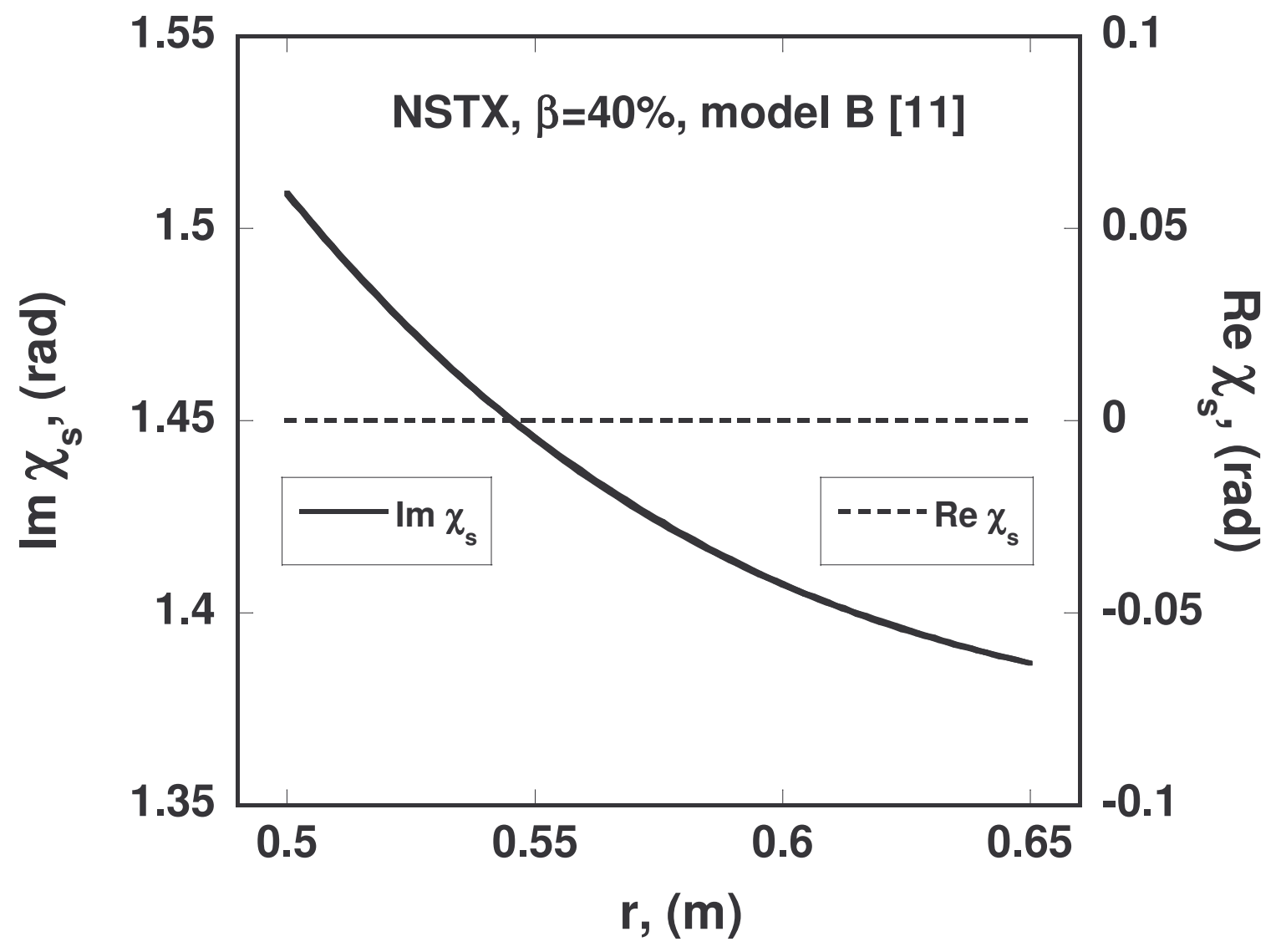

Fig. 1 


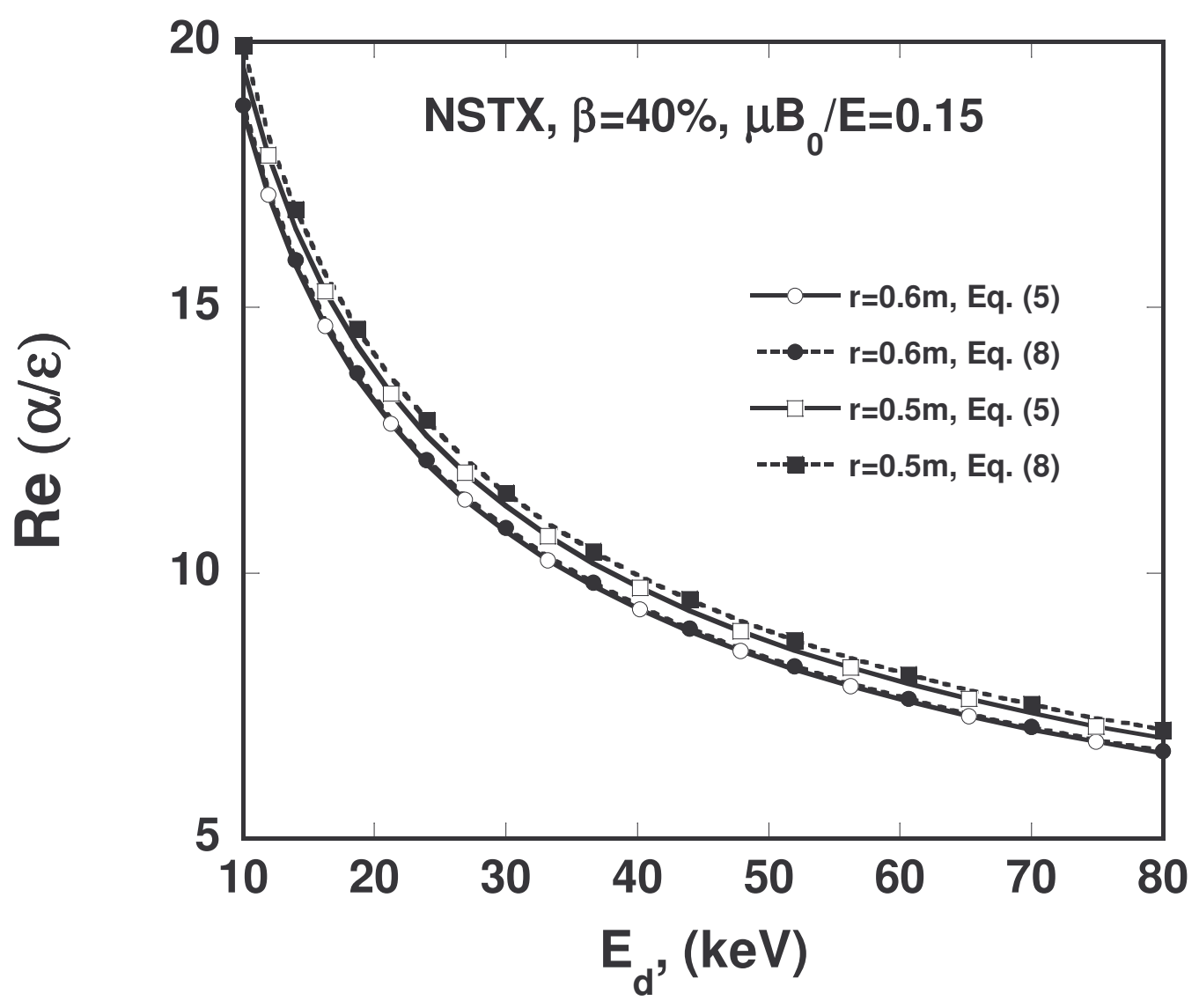

Fig. 2 


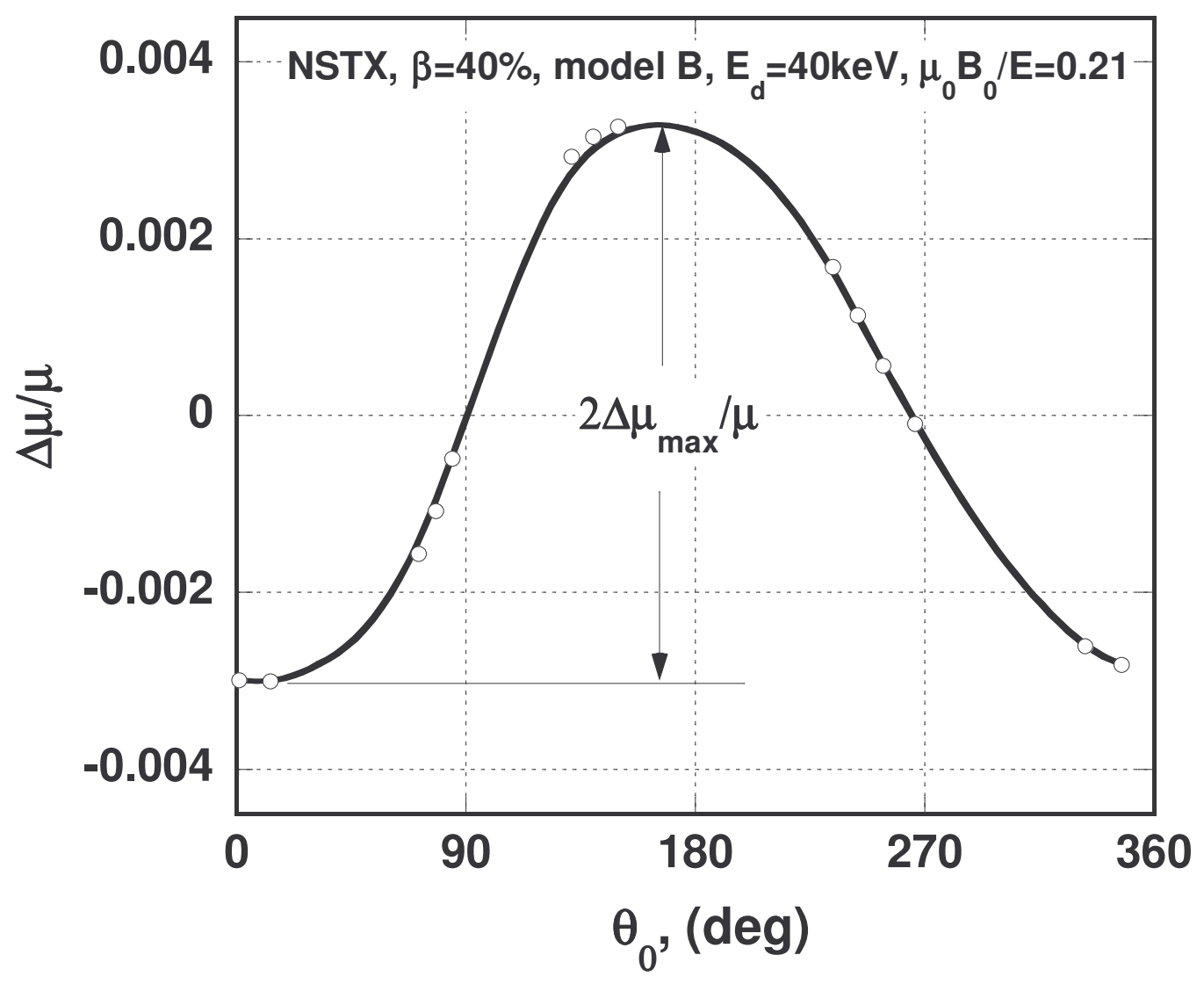

Fig. 3 


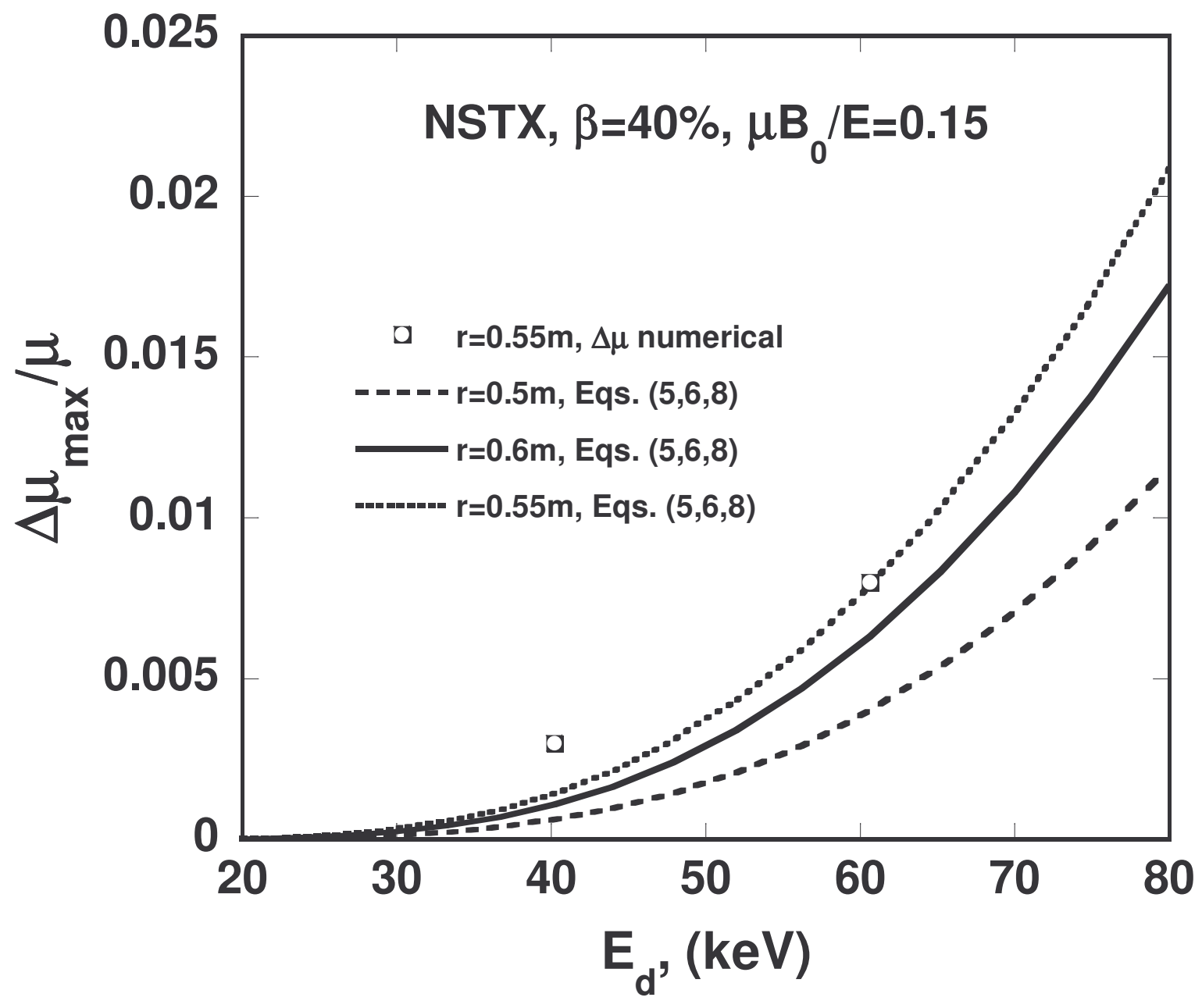

Fig. 4 


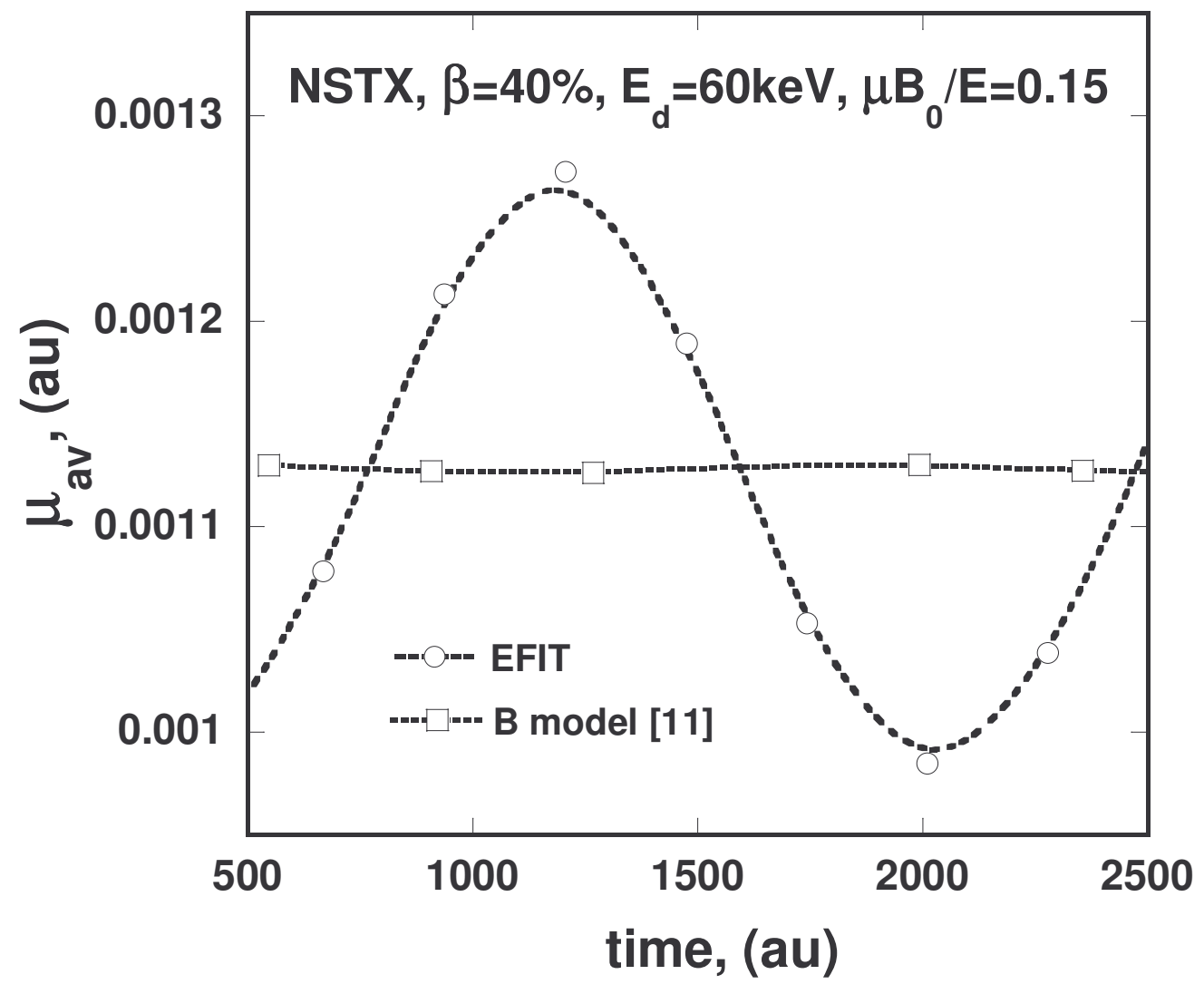

Fig. 5 


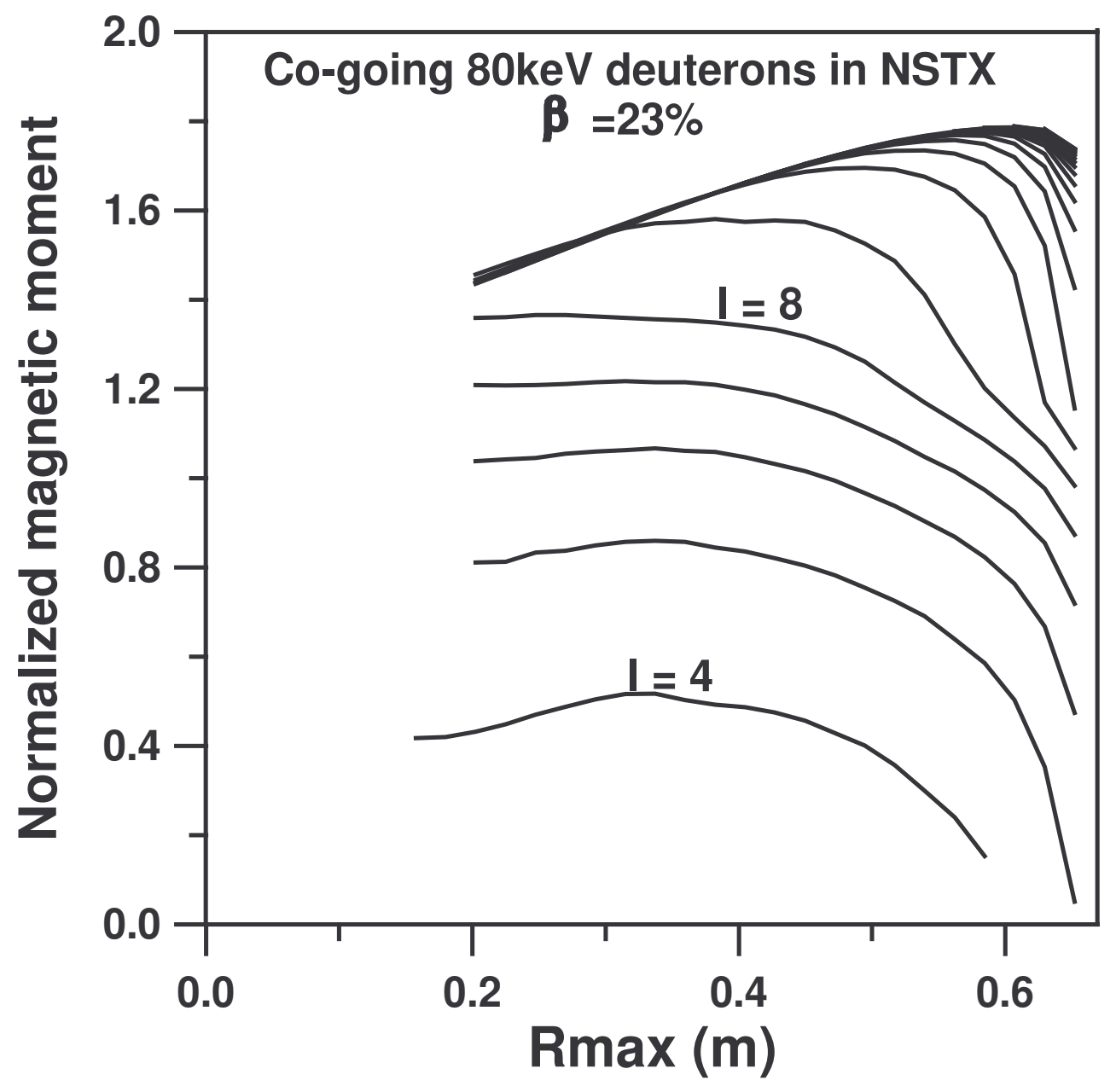

Fig. 6 

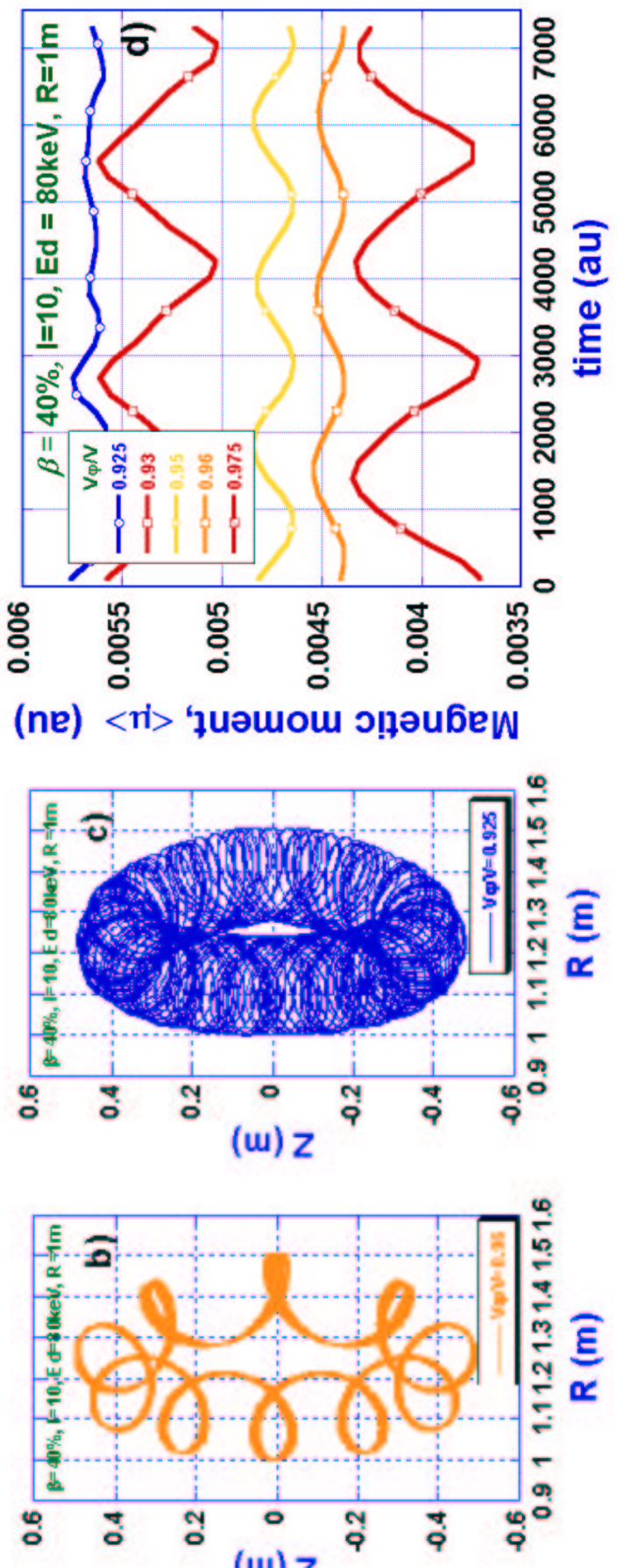

(u) $Z$

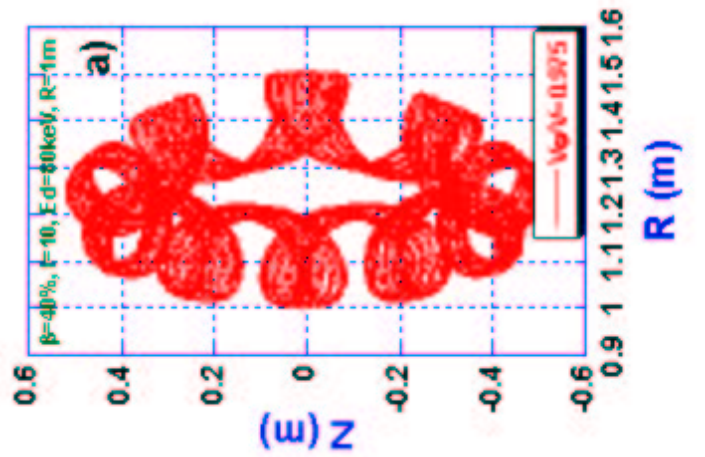



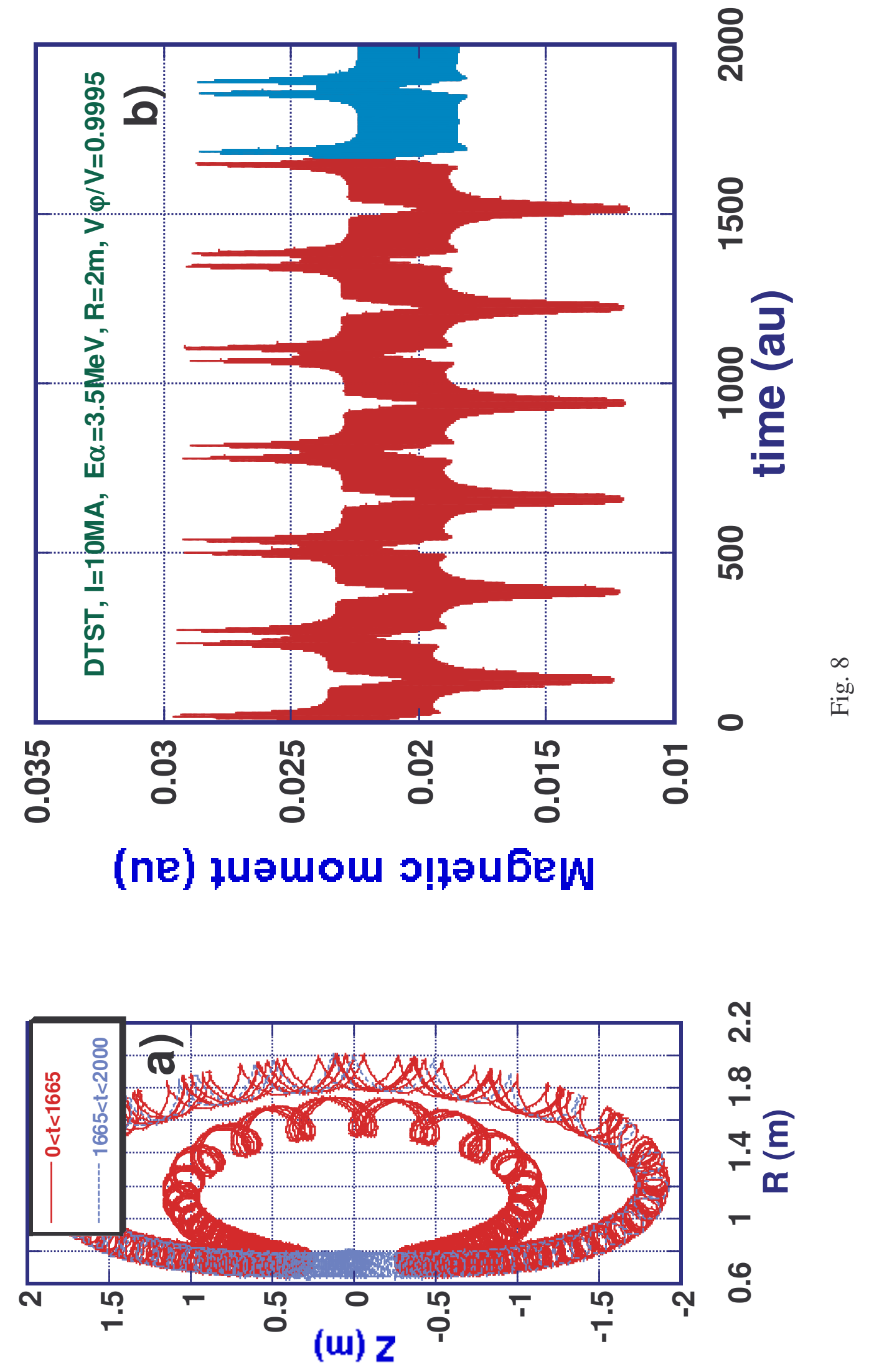


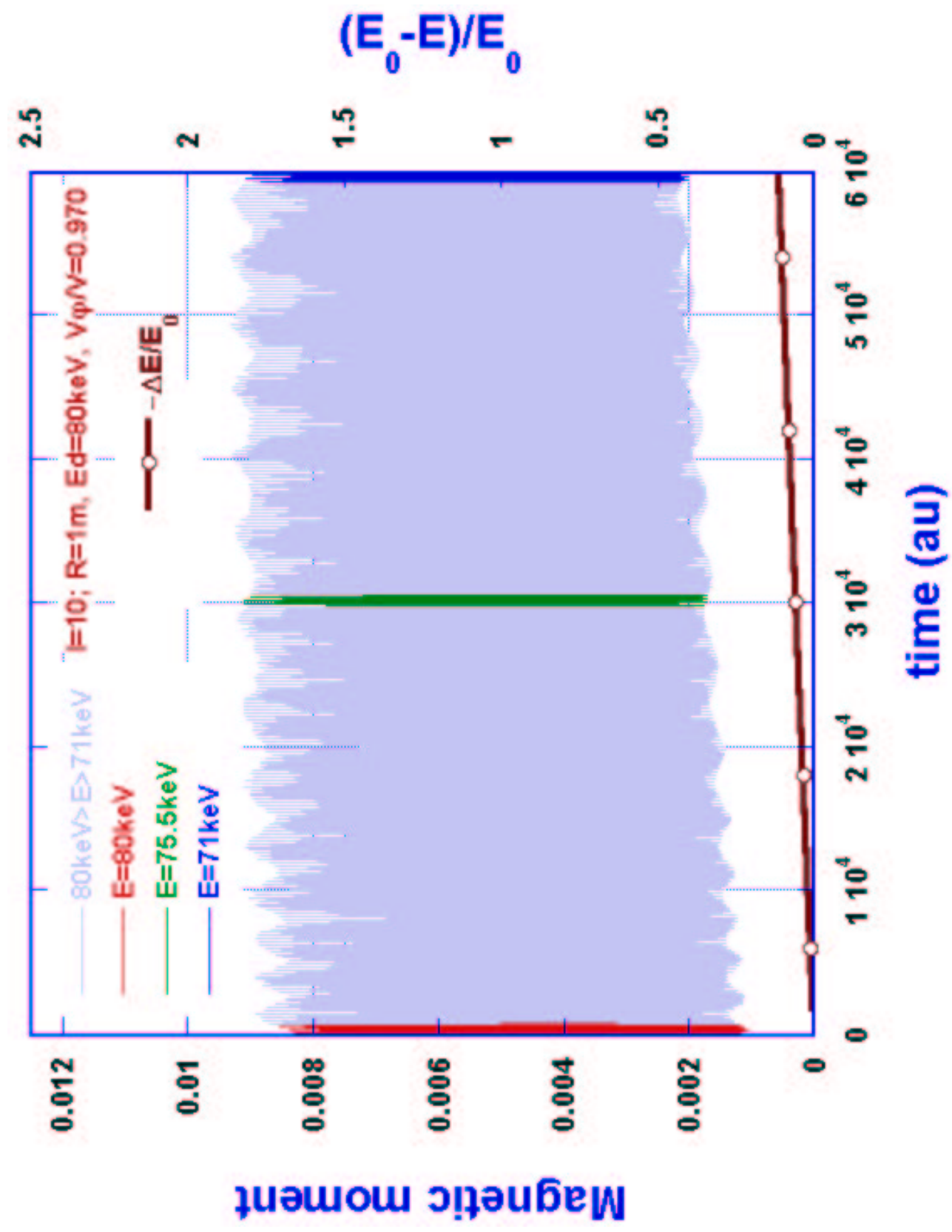

a)

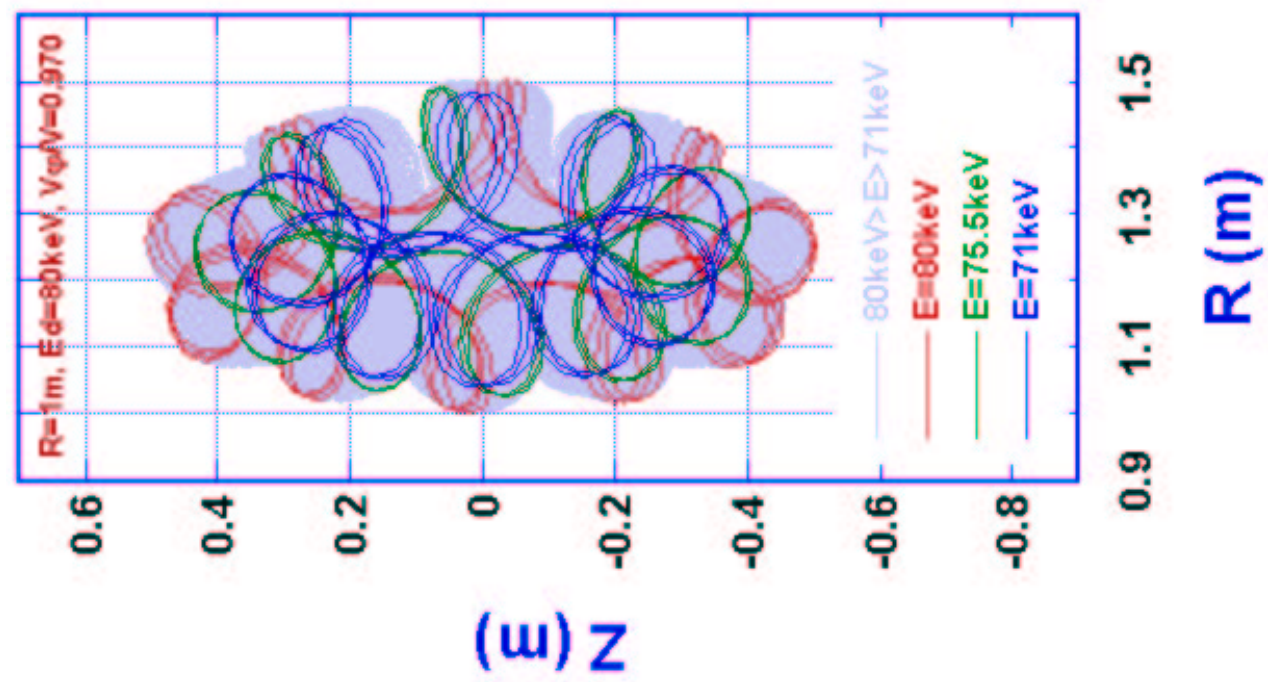




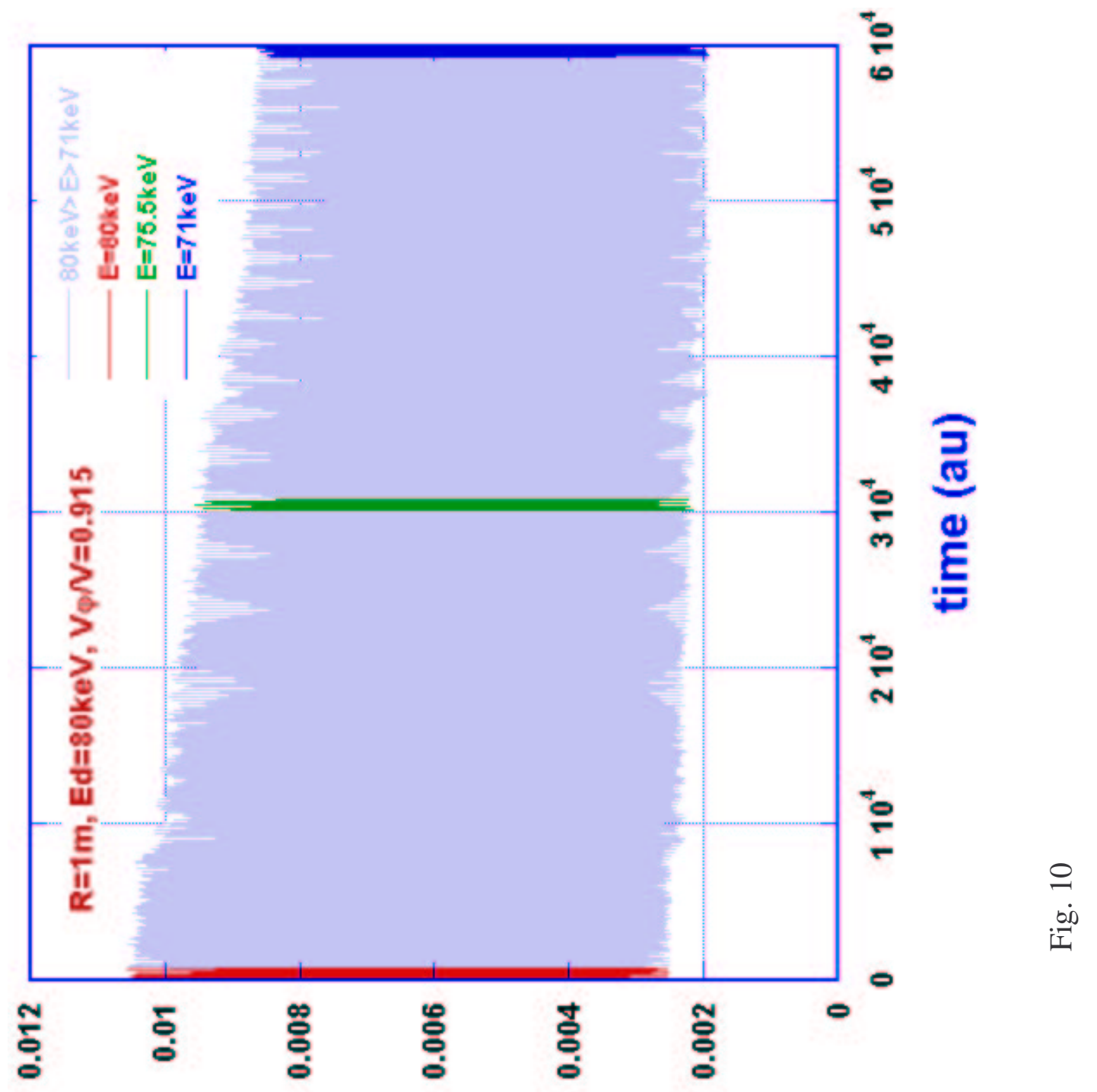

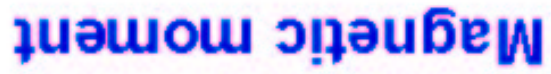

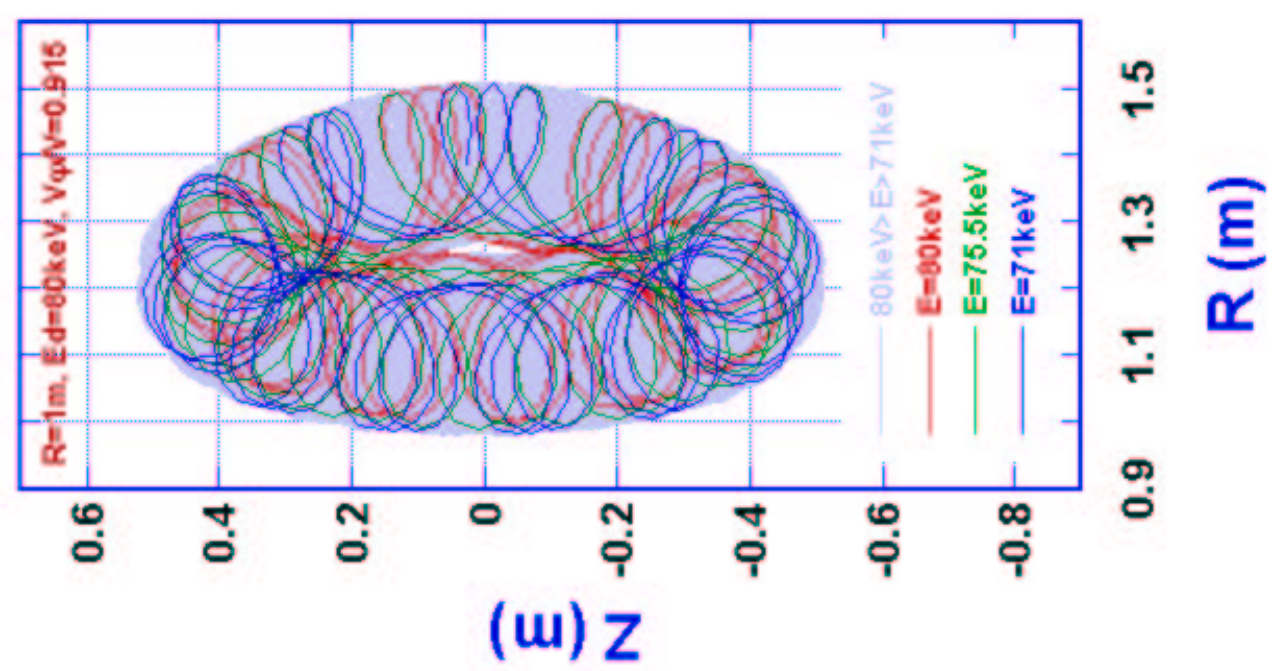




\section{External Distribution}

Plasma Research Laboratory, Australian National University, Australia

Professor I.R. J ones, Flinders University, Australia

Professor J oão Canalle, Instituto de Fisica DEQ/IF - UERJ , Brazil

Mr. Gerson O. Ludwig, Instituto Nacional de Pesquisas, Brazil

Dr. P.H. Sakanaka, Instituto Fisica, Brazil

The Librarian, Culham Laboratory, England

Library, R61, Rutherford Appleton Laboratory, England

Mrs. S.A. Hutchinson, JET Library, England

Professor M.N. Bussac, Ecole Polytechnique, France

Librarian, Max-Planck-Institut für Plasmaphysik, Germany

J olan Moldvai, Reports Library, MTA KFKI-ATKI, Hungary

Dr. P. Kaw, Institute for Plasma Research, India

Ms. P.J . Pathak, Librarian, Insitute for Plasma Research, India

Ms. Clelia De Palo, Associazione EURATOM-ENEA, I taly

Dr. G. Grosso, Instituto di Fisica del Plasma, Italy

Librarian, Naka Fusion Research Establishment, J AERI, J apan

Library, Plasma Physics Laboratory, Kyoto University, J apan

Research Information Center, National Institute for Fusion Science, J apan

Dr. O. Mitarai, Kyushu Tokai University, J apan

Library, Academia Sinica, Institute of Plasma Physics, People's Republic of China

Shih-Tung Tsai, Institute of Physics, Chinese Academy of Sciences, People's Republic of China

Dr. S. Mirnov, TRINITI, Troitsk, Russian Federation, Russia

Dr. V.S. Strelkov, Kurchatov Institute, Russian Federation, Russia

Professor Peter Lukac, Katedra Fyziky Plazmy MFF UK, Mlynska dolina F-2, Komenskeho Univerzita, SK-842 15 Bratislava, Slovakia

Dr. G.S. Lee, Korea Basic Science Institute, South Korea

Mr. Dennis Bruggink, Fusion Library, University of Wisconsin, USA

Institute for Plasma Research, University of Maryland, USA

Librarian, Fusion Energy Division, Oak Ridge National Laboratory, USA

Librarian, Institute of Fusion Studies, University of Texas, USA

Librarian, Magnetic Fusion Program, Lawrence Livermore National Laboratory, USA

Library, General Atomics, USA

Plasma Physics Group, Fusion Energy Research Program, University of California at San Diego, USA

Plasma Physics Library, Columbia University, USA

Alkesh Punjabi, Center for Fusion Research and Training, Hampton University, USA

Dr. W.M. Stacey, Fusion Research Center, Georgia Institute of Technology, USA

Dr. J ohn Willis, U.S. Department of Energy, Office of Fusion Energy Sciences, USA

Mr. Paul H. Wright, Indianapolis, Indiana, USA 
The Princeton Plasma Physics Laboratory is operated by Princeton University under contract with the U.S. Department of Energy.

\author{
Information Services \\ Princeton Plasma Physics Laboratory \\ P.O. Box 451 \\ Princeton, NJ 08543
}

Phone: 609-243-2750

Fax: 609-243-2751

e-mail: pppl_info@pppl.gov

Internet Address: http://www.pppl.gov 